\title{
Case management of childhood tuberculosis in children's hospitals in Khartoum
}

\author{
T. Osman ${ }^{7}$ and A. El Sony ${ }^{2}$
}

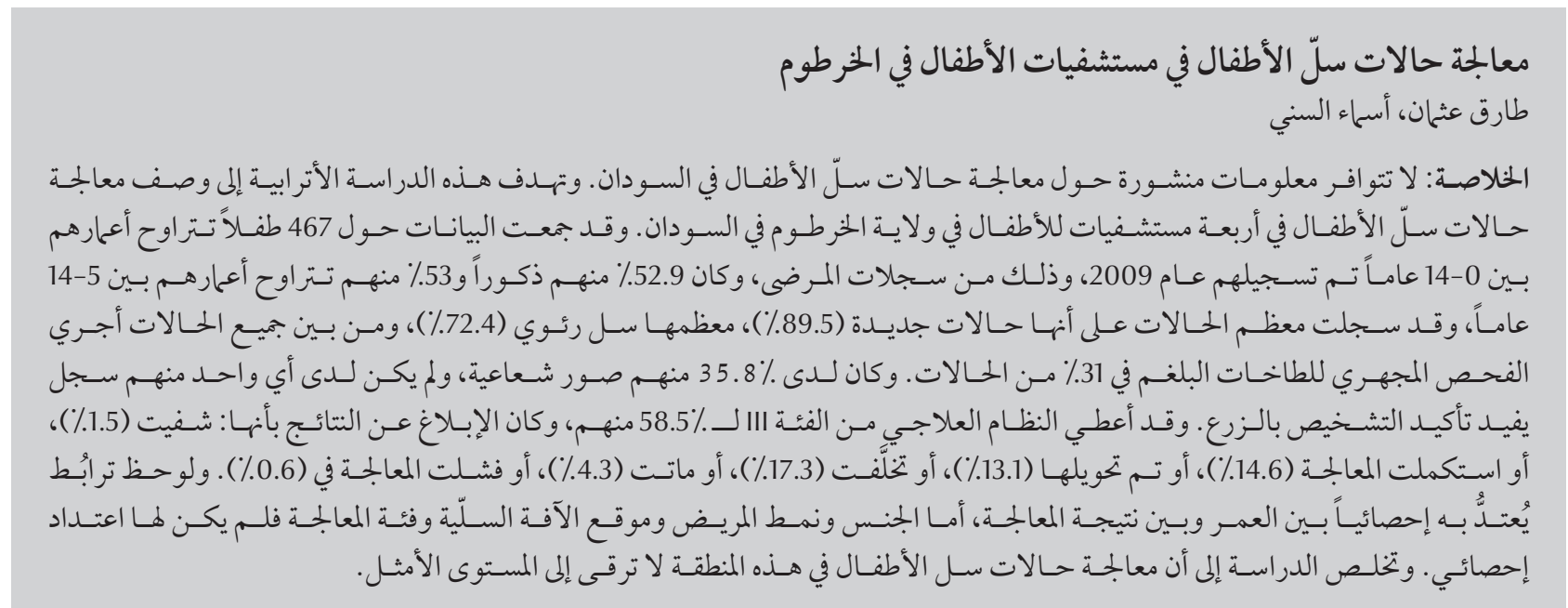

ABSTRACT No published information is available on the case management of childhood tuberculosis (TB) in Sudan. The aim of this study was to describe the case management of childhood TB in 4 children's hospitals in Khartoum State, Sudan. Data on 467 children aged 0-14 years registered in 2009 were collected from patient records; $52.9 \%$ males and $53.0 \%$ aged $5-14$ years. Most cases were registered as new cases (89.5\%) and most had pulmonary TB (72.4\%). Of all cases, 31.0\% had sputum smear microscopy done, 35.8\% had X-ray and none had a record of being culture confirmed. Category III regimen was given to $58.5 \%$. Reported outcomes were: cured (1.5\%), completed treatment (14.6\%), transferred out (13.1\%), default (17.3\%), death (4.3\%) and treatment failure $(0.6 \%)$. Age was significantly associated with treatment outcome, while sex, type of patient, site of TB and treatment category were not significant. Case management of childhood TB is suboptimal in this region.

\section{Prise en charge des cas de tuberculose chez l'enfant à I'hôpital pour enfants de Khartoum}

RÉSUMÉ II n'y a pas d'informations publiées sur la prise en charge de la tuberculose chez l'enfant au Soudan. L'objectif de la présente étude était de décrire la prise en charge de la tuberculose chez l'enfant dans quatre hôpitaux pour enfants de l'État de Khartoum (Soudan). Les données de 467 enfants âgés de 0 à 14 ans enregistrées en 2009 ont été recueillies à partir de leurs dossiers médicaux ; 52,9\% étaient des garçons et 53,0 \% étaient âgés de 5 à 14 ans. La plupart des cas étaient enregistrés comme des nouveaux cas (89,5\%) et la majorité était atteinte de tuberculose pulmonaire (72,4\%). Sur l'ensemble des cas étudiés, 31,0 \% avaient fait l'objet d'examens microscopiques de frottis d'expectoration, 35,8 \% avaient été soumis une radiographie mais aucun cas n'avait été confirmé par culture en laboratoire. Un traitement de catégorie III a été administré à 58,5\% d'entre eux. Les résultats recueillis étaient les suivants : guérison (1,5\%), traitement achevé (14,6\%), réorientation (13,1\%), abandon du traitement $(17,3 \%)$, décès $(4,3 \%)$ et échec du traitement $(0,6 \%)$. L'âge était significativement associé à l'issue du traitement, tandis que le sexe, le type de patient, le site de la tuberculose et la catégorie du traitement ne l'étaient pas. La prise en charge de la tuberculose chez l'enfant n'est pas optimale dans cette région.

'Sudan Medical and Scientific Research Institute, University of Medical Sciences and Technology, Khartoum, Sudan. (Correspondence to T. Osman: tarikod@gmail.com).

${ }^{2}$ Epidemiological Laboratory, Khartoum, Sudan.

Received: 28/07/13; accepted: 04/03/14 


\section{Introduction}

Tuberculosis (TB) kills more youths and adults than any other single infectious disease in the world (1). It is estimated that in 2010 there were 8.8 million incident cases, 12 million prevalent cases and 1.4 million deaths from TB. The greatest burden of disease is in Asia and Africa. Africa is the most affected region, with dual TB/HIV infection comprising $82 \%$ of the 1.1 million cases of TB (2).

$\mathrm{TB}$ is an important cause of morbidity and mortality in children (3). There are approximately 1 million cases of childhood TB annually (4). Estimates of disease burden suggest that children account for $11 \%$ of TB cases globally $(5,6)$. However, the proportion of TB cases that occur in children is highly variable across different countries and may reach 40\% in sub-Saharan Africa (4-8). The global burden of TB in children and its impact on the health of children are being increasingly recognized (9). In developing countries, TB is responsible for $10 \%$ of childhood hospital admissions and $10 \%$ of hospital deaths (5). Globally, it continues to exact a high toll of disease and death among children, particularly in the wake of the HIV epidemic (6).

Sudan bears $15 \%$ of the TB burden in the Eastern Mediterranean Region (10), and despite some improvements is lagging behind in progress towards achieving the Millennium Development Goals (11). In 2010 the case detection rate was 50\%. In 2009, 62\% of cases were cured, 19\% completed treatment, $3 \%$ died, $1 \%$ failed treatment, $10 \%$ defaulted and $6 \%$ were not evaluated. A best estimate for the number of HIV-positive incident TB cases in the country is 7100 (2).

The strategy of the National TB Programme (NTP) in Sudan is screening of children who are contacts through tuberculin skin testing and chest X-ray or on the basis of clinical assessment if these tests are not available. Children not found to have TB receive isoniazid preventive therapy. Children found to have TB undergo the full routine diagnostic procedures (history, chest X-ray, bacteriological confirmation of specimens by microscopy, culture and histopathology), treatment (according to diagnostic category), recording and reporting (12).

There are no published articles on the clinical characteristics, case management and treatment outcomes for childhood TB in Sudan. In Sudan, a low case detection rate, a high prevalence of adult TB cases, pervasive poverty, poor TB treatment outcomes, rampant childhood malnutrition and diagnostic challenges for case ascertainment all provide a setting for suboptimal childhood TB management. The purpose of this cohort review was to describe the characteristics, treatment outcomes and case management of childhood TB in the setting of children's hospitals in Khartoum.

\section{Methods}

\section{Design and setting}

This was a retrospective cohort review of 467 children aged $0-14$ years who were registered as TB cases and received treatment from January to December 2009. All records of childhood TB cases during this period were reviewed. The guidelines for management of childhood TB in Sudan were changed in 2010. However, since the cohort of 2010 was not complete by the start of the study, a decision was made to review the cohort study for 2009. The cohort for 2009 was managed according to the 2006 guidelines of the National manual for the management of childhood tuberculosis in Sudan (12). The 2006 manual provides diagnostic and management guidelines that follow the guidelines outlined in the World Health Organization's (WHO) Guidance for national tuberculosis programmes on the management of tuberculosis in children (13).

Children with TB are diagnosed and registered for treatment at children's hospitals in Sudan. The TB register and follow-up treatment cards are supervised by the paediatricians and coordinators of the NTP management units at these hospitals. Treatment cards contain patient demographics, TB clinical characteristics and follow-up progress on management. The study was conducted at 4 tertiary care children's hospitals (Jaafar Ibn Ouf Children's Hospital, Omdurman Children's Hospital, Ahmed Gasim Children's Hospital and Elbuluk Children's Hospital) in Khartoum State, Sudan.

\section{Data collection and analysis}

The data sources were the TB registry and treatment cards. The primary variables of interest as defined by the national guidelines were: type of patient, site of $\mathrm{TB}$, category of regimen and treatment outcome. The secondary variables of interest were: age, sex, type of investigations (chest X-ray, smear microscopy, culture), pretreatment weight measurement, HIV counselling, HIV testing and HIV status.

The data were recorded in a specially designed, structured questionnaire. All inconsistencies were resolved by referring to the records. Where no patient data for the variable were recorded in the data sources, it was classified as unknown.

\section{Definitions}

The definitions used were in line with similar studies and standard WHO definitions (13).

- New case: TB patient who has never had treatment for TB or has taken anti-TB drugs for $<1$ month.

- Relapsed: TB patient who had been declared cured or whose treatment had been completed by a physician, but who reports back to the health 
service and is now found to be sputum smear-positive.

- Transferred in: TB patient who has been received for treatment into a TB unit after starting treatment in another unit where s/he had been registered).

- Treatment after default: TB patient who has received anti-TB treatment for at least 1 month from any source and has returned to treatment after having defaulted.

- Other: TB patient who does not fit into the above-mentioned types.

- Cured: TB patient who is sputum smear-negative in the last month of treatment and on at least 1 previous occasion

- Completed treatment: TB patient who has completed treatment but who does not meet the criteria to be classified as cured or treatment failure.

- Default: TB patient whose treatment was interrupted for 2 consecutive months or more.

- Died: TB patient who dies for any reason during the course of treatment.

- Treatment failure: TB patient who is sputum smear positive at 5 months or later after starting treatment.

- Transferred out: TB patient who has been transferred to another recording and reporting unit and for whom the treatment outcome is not known.

\section{Ethical considerations}

The protocol was reviewed and granted clearance by the ethics committee of the Khartoum State Ministry of Health and the NTP Coordinator of Khartoum State. Final consent was obtained from the directors of the 4 hospitals to access the data archives. Consent was not required from parents as this study involved a review of records.

\section{Data analysis}

The data were analysed using SPSS for Windows, version 16. Basic descriptive analysis and calculation of proportions were performed. Chi-squared tests and Fisher exact test were performed for comparison of categorical data. Statistical significance was defined as $P<0.05$. The statistical power of the study was $95 \%$.

\section{Results}

\section{Characteristics of children with TB}

\section{Age and sex characteristics}

Table 1 shows the demographic and clinical characteristics of children with TB. Of the 467 cases registered and receiving treatment at the children's hospitals, 247 (52.9\%) were males and 220 (47.1\%) were females. The M:F ratio was 1.2:1. There were fewer children in the $0-4$ year age category $(46.7 \%)$ than in the 5-14 years age category (52.7\%); 3 children did not have their age recorded. The median age was 60 months [interquartile range (IQR) 24-108 months].

\section{Childhood TB characteristics}

Most cases $(418,89.5 \%)$ were new, 5 children $(1.1 \%)$ were classified as relapsed cases, 13 (2.8\%) as transferredin, $9(1.9 \%)$ as treated after default and $1(0.2 \%)$ was classified as other; in 21 cases $(4.5 \%)$ the type of patient was unknown. There were more pulmonary TB (72.4\%) than extrapulmonary TB cases $(27.4 \%)$; in 1 case $(0.2 \%)$ the site of TB was not recorded. Of the children 9.2\% had smear-positive pulmonary TB and $26.0 \%$ had smear-negative pulmonary TB; in $64.8 \%$ of children the smear status of pulmonary TB was unknown. The specific types of extrapulmonary TB were not recorded. Radiography results were positive in $30.0 \%$, negative in $5.8 \%$ and unknown for $64.2 \%$ of children. None of the cases had any record of culture being done. Pretreatment weight measurement was taken for $61.0 \%$ of children, but was unknown for 39.0\%. Category III and category I regimens were given to $58.5 \%$ and $36.4 \%$ of cases respectively; there was no record of the treatment category for $1.9 \%$. A total of $42.8 \%$ cases had received the treatment regimen for their disease category, but $7.3 \%$ did not receive the recommended regimen. In $49.9 \%$ of children it could not be determined whether the correct category of regimen was administered. The median duration of treatment was 223 days (IQR: 202-241 days).

\section{Treatment outcomes}

Information on treatment outcome was available for 240 (51.4\%) cases. Overall $16.1 \%$ had a favourable treatment outcome; $1.5 \%$ were cured and $14.6 \%$ completed treatment. The unfavourable treatment outcomes were: default (17.3\%), death (4.3\%) and treatment failure $(0.6 \%)$. There were $13.1 \%$ of cases recorded as transferred out. The duration of treatment was defined for 48 children $(64.0 \%)$ with a favourable outcome.

\section{TB/HIV}

HIV counselling was provided to 24 (5.1\%) of the cohort. A total of 57 children (12.2\%) had had an HIV test and only $2(0.4 \%)$ were HIV positive; both were males, were from both age groups, were new cases, had smear-negative pulmonary $\mathrm{TB}$, received category III regimen and had no record of receiving counselling, anti-retroviral therapy (ART) or co-trimoxazole preventive therapy. One defaulted and the other was transferred out.

\section{Association between children's characteristics and treatment outcomes}

Table 2 shows the association between the outcome of treatment and sex, age, type of patient, site of TB and treatment category. Considering the treatment outcomes and whether they were favourable or unfavourable, the associations between treatment outcome with sex, type of patient, site of TB and treatment category were all non-significant $(P=0.891, P=0.306, P=0.108$ and $P$ 

Table 1 Demographic and clinical characteristics of children with tuberculosis (TB)
$(n=467)$

Variable

Demographic characteristics

\section{Age (years)}

$0-4$

5-14

218

246

Unknown

No.

$\%$

Sex

Male

247

52.9

Female

220

47.1

\section{TB characteristics}

\section{Type of patient}

New

418

89.5

Relapsed

Transferred in

Treatment after default

Other

Unknown

Site of $T B$

Pulmonary TB

Smear-positive

Smear-negative

Unknown

Extrapulmonary TB

Unknown

1

\section{Investigations}

\section{Smear microscopy}

Done

Unknown

Radiography

Positive

Negative

Unknown

\section{Culture}

Unknown

\section{Pretreatment weight measurement}

Recorded

Not recorded

\section{Treatment}

\section{Treatment category}

I

II

III

Unknown

9

\section{Category regimen}

As recommended

Not as recommended

Unknown
$=0.298$ respectively). However, significantly more cases in the 5-14 year age group had favourable outcomes (52.7\% versus $47.3 \%, P=0.002$ ).

\section{Discussion}

This was the first hospital-based study to review the characteristics, treatment outcomes and case management of childhood TB in children's hospitals in Sudan. It was a review of management from patients' records. It is critical to evaluate adherence to NTP policies for childhood TB management and the effectiveness of the management of childhood TB by hospital paediatricians. The study highlights pertinent points for the review of childhood TB management in children's hospitals.

For comparisons with other studies we searched the literature with a primary focus on research from Africa. We used the following search terms: childhood TB, paediatric TB, paediatric $\mathrm{TB}$, standard case management, case management, treatment outcomes, characteristics, investigations, diagnosis, developing countries, Africa, Asia and Eastern Mediterranean Region to construct search strategies. We searched PubMed, HINARI, WHO website and Google Scholar to identify the freely available, scholarly and scientific references on the subject matter. The oldest reference was from 2002.

\section{Reporting and recording}

Children registered and managed in children's hospitals should have complete TB registers and treatment cards. Five key elements of childhood TB standard case management allow for valid assessment of the quality of case management. These are: pretreatment weight measurement, type of patient, site of TB, treatment category and treatment outcome. In this cohort, recording and reporting of type of patient, site of TB and category of treatment regimen were adequate but there were 


\begin{tabular}{lrc}
\hline $\begin{array}{l}\text { Table 1 Demographic and clinical characteristics of children with tuberculosis (TB) } \\
(\boldsymbol{n}=\mathbf{4 6 7 )} \text { (concluded) }\end{array}$ & No. \\
\hline Variable & 7 & 1.5 \\
Treatment outcome & 68 & 14.6 \\
$\quad$ Cured & 3 & 0.6 \\
Completed treatment & 20 & 4.3 \\
Failure & 81 & 17.3 \\
Died & 61 & 13.1 \\
Default & 227 & 48.6 \\
Transferred out & & \\
Unknown & & \\
TB/HIV & 24 & 5.1 \\
HIV counselling & 443 & 94.9 \\
Yes & & \\
Unknown & 57 & 12.2 \\
HIV testing & 410 & 87.8 \\
Yes & & \\
Unknown & 2 & 0.4 \\
HIV status & 39 & 8.4 \\
Seropositive & 426 & \\
Seronegative & & \\
Unknown & & \\
\hline
\end{tabular}

inadequacies in reporting of pretreatment weight measurement, investigations, type of pulmonary TB and treatment outcomes. The recording and reporting system identifies patients who are failing therapy (14). Inadequacies in recording and reporting have been reported elsewhere, particularly with regards to treatment outcomes $(15,16)$. Inadequate reporting of weight raises ethical concerns. For example, children in Malawi receiving anti-TB treatment were reported to have had inappropriate and low drug dosages (8). Weight is an indicator for response to treatment. Pretreatment weight measurement is important and should be common practice in Sudan, where childhood malnutrition is rampant. The outcome of TB treatment in children is frequently not reported. The highest reported rate for not reporting treatment outcome was $87 \%$ in Pakistan (17). This may reflect unfamiliarity with the recording and reporting process, lack of training or poor follow-up by treatment providers, or poor level of commitment by practitioners, workload and limited experience (17).

Because of poor recording of the date of symptoms and date of initiation of treatment, we were unable to measure the total delay in initiating treatment. We hypothesize that there was diagnostic delay, as a result of underutilization of investigations, which would have resulted in prolonged total delay in treatment initiation in a major proportion of the cohort. Diagnostic delay increases the severity of infection, development of complications, prolonged infectivity in the community and leads to higher mortality $(18,19)$. Other reported factors that may lead to delayed treatment include service factors (lack of diagnostic facilities, lack of trained staff, lack of good quality services, lack of effective supervision, differences in differential diagnoses between physicians reflecting varying degrees in clinical suspicion); living far from a diagnostic and/or treatment facility; individual variations in the perception of disease; and recall bias leading to poor measurement of date of onset of symptoms $(20,21)$.

Most childhood cases are new cases and are therefore given category I and III treatment regimens. In this cohort we attempted to determine whether each case received the correct category regimen. The correct regimen was determined if the following were recorded: category regimen, site of $\mathrm{TB}$, type of patient and smear result on diagnosis. Because of inadequacies in reporting and recording in almost half the cases it could not be determined if the correct regimen was given. In 7.3\% of the cases the correct regimen was not given as recommended when crosschecking the category regimen with site of TB, type of patient and smear result on diagnosis. We found no data that reported on the proportion of children who received the correct regimen.

Recording and reporting of dates of treatment initiation and completion was mostly inadequate. The duration of treatment was measured for only 48 new cases with a favourable treatment outcome, in which the dates of treatment initiation and completion were recorded. It was observed that some cases had received less than the recommended duration of treatment, while some cases appear to have had an extended period of treatment. The reasons for receiving less than the recommend duration of treatment are unclear. It can be postulated that those with a longer duration of treatment had more severe disease or were receiving treatment on an irregular basis. The factors for prolonged treatment should be investigated. The median duration of treatment among this cohort (223 days; IQR: 202-241 days) was longer than the median duration reported for a cohort of patients in India (182 days; IQR: 178-187 days) whose outcome was treatment completion with the treatment regimen for new cases (22). 


\begin{tabular}{|c|c|c|c|c|c|}
\hline \multirow[t]{2}{*}{ Variable } & \multicolumn{2}{|c|}{ Favourable outcome } & \multicolumn{2}{|c|}{$\begin{array}{l}\text { Unfavourable } \\
\text { outcome }\end{array}$} & \multirow[t]{2}{*}{$P$-value } \\
\hline & No. & $\%$ & No. & $\%$ & \\
\hline \multicolumn{6}{|l|}{ Sex } \\
\hline Male & 39 & 42.2 & 53 & 57.6 & \multirow{2}{*}{0.891} \\
\hline Female & 36 & 41.4 & 51 & 58.6 & \\
\hline \multicolumn{6}{|l|}{ Age (years) } \\
\hline $0-4$ & 26 & 30.2 & 60 & 69.8 & \multirow{2}{*}{0.002} \\
\hline $5-14$ & 49 & 52.7 & 44 & 47.3 & \\
\hline \multicolumn{6}{|l|}{ Type of patient } \\
\hline New case & 68 & 43.3 & 89 & 56.7 & \multirow{2}{*}{0.306} \\
\hline Other & 7 & 31.8 & 15 & 68.2 & \\
\hline \multicolumn{6}{|l|}{ Site of $T B$} \\
\hline Pulmonary TB & 52 & 38.5 & 83 & 61.5 & \multirow{2}{*}{0.108} \\
\hline Extrapulmonary TB & 23 & 52.3 & 21 & 47.7 & \\
\hline \multicolumn{6}{|l|}{ Treatment category } \\
\hline I & 13 & 56.5 & 10 & 43.5 & \multirow{3}{*}{0.298} \\
\hline II & 2 & 33.3 & 4 & 66.7 & \\
\hline III & 60 & 40.0 & 90 & 60.0 & \\
\hline
\end{tabular}

\section{Sputum microscopy, radiology} and culture

The underutilization of diagnostic methods is reported in both underdeveloped and developed regions $(17,23,24)$. The use of a chest X-ray has been shown to be helpful in detecting changes suggestive of $\mathrm{TB}$ in children with pulmonary $\mathrm{TB}$ and has been supported internationally (17). Our study has shown that chest radiography was underutilized in this cohort. In Malawi, over $80 \%$ of the childhood TB cases had radiography findings consistent with TB (25). Possible explanations for the low rate of microbiological confirmation include failure to report a proportion of cultureconfirmed cases and different spectra of disease or difficulties in obtaining specimens, especially in smaller children and infants (26). It could also be related to less aggressive investigation in childhood TB cases diagnosed following an adult index case (26).

The ratio of pulmonary to extrapulmonary $\mathrm{TB}$ according to the $\mathrm{WHO}$ is 1:3 (5). A greater proportion of children in our study had smear-negative pulmonary TB (smear-positive to smear-negative ratio 1:2.7). The majority of children had no record of sputum smear examinations on initiation of treatment, radiography and none had a record of culture done for microbiological confirmation. Other studies have shown that only $10 \%-15 \%$ of sputum samples reveal acid-fast bacilli on microscopy $(6,27)$ and even culture remains negative in around $70 \%$ of cases with probable TB (6). A study in South Africa indicated missed opportunities for treatment in $64 \%$ of a cohort of 596 cases (9). It would be expected that within children's hospitals smear microscopy and radiography would be routinely conducted. In more than half our cohort, there was no record of radiography, indicating underutilization of radiography. Diagnostic misclassification may occur in 15\%-20\% of childhood TB cases (22). This may lead to misclassifying the case under the wrong treatment category. Other forms of TB were designated as extrapulmonary TB without indicating the specific type of extrapulmonary TB. There is a need to emphasize the importance of documenting the type of extrapulmonary TB. It is likely that the diagnosis of pulmonary $\mathrm{TB}$ and extrapulmonary $\mathrm{TB}$ was based on history, clinical presentation and on a presumptive basis in the absence of bacteriological confirmation $(8,23)$.

\section{Treatment outcomes}

Children tend to have favourable TB outcomes in general (13). The proportion of children with successful outcome is an indicator of the quality of $\mathrm{TB}$ case management. The treatment outcomes were poor for this cohort. A low treatment success rate, high rates of unfavourable outcomes and failure to report outcomes in a significant proportion of cases is a reflection of poor case management. The reasons for this remain unclear, but it has been reported that case management can be affected by issues related to accurate diagnosis, lack of resources and quality control (16). The overall success rate in our cohort was $16.1 \%$, which is below the WHO recommended target of $85 \%$ and lower than rates reported from other countries $(23,28)$. It is also lower than the success rate for adult TB in Sudan (10). Favourable outcomes among children have been reported to range from $45 \%$ to $95 \%(8,15,22,23,29,30)$. Of the 75 children with favourable treatment outcomes in our study, only 48 (64.0\%) had the duration of treatment defined. The high default rate is above the Eastern Mediterranean Region estimate of $8 \%$ (28). However, as these were tertiary care referral hospitals, it is possible that patients abandoned treatment because they were living far from the hospitals or were referred from distant regions. This may partly explain the high transferredout rate. There is a need to identify the determinants of default and ways to improve it. In this cohort older children had significantly more favourable outcomes. Young age is associated with risk of developing disease, and death is more 
common among young children aged 5 years and under $(5,6,8,15)$.

\section{TB/HIV}

Africa bears about $78 \%$ of the total burden of TB/HIV infections in the world (31). The coinfection rate among children exceeds $50 \%$ in some African settings (6). Sudan is beginning to experience the dual epidemics of TB and HIV (32). Sudan is the country most affected by AIDS in the Eastern Mediterranean Region, with an estimated 512000 cases, and is experiencing a generalized epidemic with HIV prevalence more than $1 \%$ in the general population aged 15-49 years. The prevalence of TB/HIV among adults was estimated at $4.8 \%$ in 2001 (11). Programme guidance for 2006 requires HIV counselling and testing for TB patients with symptoms and/ or signs of HIV-related conditions and in TB patients having a history suggestive of high risk of HIV exposure. There is no record that HIV testing was done with counselling in this cohort. Only 2 cases were HIV positive out of the 57 cases tested. Both TB cases had no record of receiving ART. Data from the WHO indicate that $41 \%$ of notified TB patients were tested for HIV in Sudan and $6.2 \%$ were found to be HIV positive (2). Of these $58 \%$ and $54 \%$ were started on co-trimoxazole preventive therapy and ART respectively (2). This indicates that access to co-trimoxazole prevention and ART is limited. We could not evaluate the association between HIV status and treatment outcome because of the small number of HIV positive cases.
The strengths of this study is that it is the first study in Sudan to provide insight into TB case management in children's hospitals and it has identified gaps in standard case management of childhood TB. The major limitations were that the study was retrospective in nature; the results cannot be generalized to non-hospital settings or private clinics, and reporting and recording was suboptimal. Although the findings are valid only to management of childhood TB in these hospitals, there is potential information bias and diagnostic misclassification bias due to missing data. Other research publications on childhood TB from developing countries have presented findings from hospital, regional or national data $(8,15,17,22,23,25)$.

In conclusion, we reported on case management of childhood TB in Sudan from information recorded in files to assess reporting and recording, investigations conducted, management and treatment outcomes. Inadequacies in reporting and recording, underutilization of diagnostic investigations and poor treatment outcomes indicated suboptimal case management of childhood TB in our children's hospitals. The implications are failure to achieve target levels for cure, relapse of disease and evolution of drug resistant TB. Paediatricians have an important public health responsibility for treating childhood TB in hospitals. There needs to be effective collaboration between the NTP and paediatricians in order to decentralize the demonstration sites to district hospitals and below. Decentralization may improve efficiency and quality of services within the context of local circumstances. Improving case management requires that all children are properly evaluated for $\mathrm{TB}$, receive standard treatment regimens and receive proper supervision for adherence to treatment. Proper reporting and recording is critical and needs to be enforced as it improves the standard of care and allows for accurate systematic analysis. Operational research is needed to investigate barriers to standard case management and factors contributing to suboptimal management and to develop strategies for improvement of case management in children's hospitals. The ultimate goal is to have a programme following standard case management guidelines at both clinical and programme level.

\section{Acknowledgements}

We appreciate the support of the Sudan NTP, Khartoum State TB Coordinator, directors of the children's hospitals and the paediatricians for providing ethical clearance and access to patient files. We also acknowledge the technical assistance and manuscript revision provided by Dr Amjad Faried, Dr Thuwaiba Ali and Dr Adil Babiker.

Funding: Funding for this research was provided by the Graduate College of the University of Medical Sciences and Technology.

Competing interests: None declared

\section{References}

1. Tuberculosis Working Group Tuberculosis control programming for POVs. Facilitator's manual. Washington (DC): CORE Group; 2006 (http://www.coregroup.org/storage/documents/Workingpapers/TB_Curriculum_4-8-06_v2.pdf, accessed 25 April 2014).

2. Global tuberculosis control 2011. Geneva: World Health Organization; 2011 .

3. Nelson LJ, Wells CD. Global epidemiology of childhood tuberculosis. Int J Tuberc Lung Dis. 2004 May;8(5):636-47. PMID:15137548
4. Swaminathan S, Rekha B. Pediatric tuberculosis: global overview and challenges. Clin Infect Dis. 2010 May 15;50(s3) Suppl 3:S184-94. PMID:20397947

5. Tahmeed A, Farzana S, Shamsir A, Sayera B, Asif M, Khurshid $\mathrm{AH}$, et al. Childhood tuberculosis: a review of epidemiology, diagnosis and management. Infect Dis J Pakistan. 2008; 17(2):52-60.

6. Newton SM, Brent AJ, Anderson S, Whittaker E, Kampmann B, Paediatric TB. Paediatric tuberculosis. Lancet Infect Dis. 2008 Aug;8(8):498-510. PMID:18652996 
7. Walls T, Shingadia D. Global epidemiology of paediatric tuberculosis. J Infect. 2004 Jan;48(1):13-22. PMID:14667788

8. Muñoz-Sellart M, Yassin MA, Tumato M, Merid Y, Cuevas LE. Treatment outcome in children with tuberculosis in southern Ethiopia. Scand J Infect Dis. 2009;41(6-7):450-5. PMID:20001277

9. Schaaf HS, Marais BJ, Whitelaw A, Hesseling AC, Eley B, Hussey GD, et al. Culture-confirmed childhood tuberculosis in Cape Town, South Africa: a review of 596 cases. BMC Infect Dis. 2007;7(1):140. PMID:18047651

10. A continuing commitment to save lives: a report on WHO collaborative programmes with the Government of Sudan and partners 2008-2009 in Sudan. Khartoum, Sudan: World Health Organization; 2009 (http://www.abhatoo.net.ma/ content/download/21681/397414/version/1/file/continuing commitment_save_lives_Sudan.pdf, accessed 12 May 2014).

11. Progress report on achievement of the Millennium Development Goals. Fifty-eighth session of the Regional Committee for the Eastern Mediterranean. Agenda item 2 (d). Cairo: WHO Regional Office for the Eastern Mediterranean; 2010 (EM/ RC58/INF.DOC.3) http://applications.emro.who.int/docs/ RC_technical_papers_2011_inf_doc_3_13992.pdf?ua=1, accessed 25 April 2014).

12. National manual for the management of childhood tuberculosis in Sudan. Khartoum: Federal Ministry of Health; 2008.

13. Guidance for national TB programmes on the management of TB in children. Geneva: World Health Organization; 2006.

14. Tuberculosis Coalition for Technical Assistance. International Standards for Tuberculosis Care (ISTC). The Hague: Tuberculosis Coalition for Technical Assistance; 2006.

15. Harries AD, Hargreaves NJ, Graham SM, Mwansambo C, Kazembe P, Broadhead RL, et al. Childhood tuberculosis in Malawi: nationwide case-finding and treatment outcomes. Int J Tuberc Lung Dis. 2002 May;6(5):424-31. PMID:12019918

16. Safdar N, Hinderaker SG, Baloch NA, Enarson DA, Khan MA, Morkve O. Childhood tuberculosis deskguide and monitoring: an intervention to improve case management in Pakistan. BMC Health Serv Res. 2011;11(1):187. PMID:21831308

17. Safdar N, Hinderaker SG, Baloch NA, Enarson DA, Khan MA, Morkve O. Are children with tuberculosis in Pakistan managed according to National programme policy guidelines? A study from 3 districts in Punjab. BMC Res Notes. 2010;3(1):324. PMID:21114868

18. Marais BJ, Obihara CC, Warren RM, Schaaf HS, Gie RP, Donald PR. The burden of childhood tuberculosis: a public health perspective. Int J Tuberc Lung Dis. 2005 Dec;9(12):1305-13. PMID:16466051

19. Hui-Ping Lin. Chung-Yeh Deng, Pesus Chou. Diagnosis and treatment delay among pulmonary TB patients identified using the Taiwan reporting enquiry system, 2002-2006. BMC Public Health. 2009;9(55)

20. Basnet R, Hinderaker SG, Enarson D, Malla P, Mørkve O. Delay in the diagnosis of tuberculosis in Nepal. BMC Public Health. 2009;9(1):236. PMID:19602255

21. Maamari F. Case-finding tuberculosis patients: diagnostic and treatment delays and their determinants. East Mediterr Health J. 2008 May-Jun;14(3):531-45. PMID:18720617

22. Satyanarayana S, Shivashankar R, Vashist RP, Chauhan LS, Chadha SS, Dewan PK, et al. Characteristics and programmedefined treatment outcomes among childhood tuberculosis (TB) patients under the national TB programme in Delhi. PLoS ONE. 2010;5(10):e13338. PMID:20967279

23. Mtabho CM, Irongo CF, Boeree MJ, Aarnoutse RE, Kibiki GS. Childhood tuberculosis in the Kilimanjaro region: lessons from and for the TB programme. Trop Med Int Health. 2010 May;15(5):496-501. PMID:20230572

24. Nelson LJ, Schneider E, Wells CD, Moore M. Epidemiology of childhood tuberculosis in the United States, 1993-2001: the need for continued vigilance. Pediatrics. 2004 Aug;114(2):33341. PMID:15286213

25. Weismuller MM, Graham SM, Claessens NJ, Meijnen S, Salaniponi FM, Harries AD. Diagnosis of childhood tuberculosis in Malawi: an audit of hospital practice. Int J Tuberc Lung Dis. 2002 May;6(5):432-8. PMID:12019919

26. Abubakar I, Laundy MT, French CE, Shingadia D. Epidemiology and treatment outcomes of childhood TB in England and Wales. Arch Dis Child. 2008;93:1017-21. PMID:18562450

27. Ait-Khaled N, Alarcon E, Armengol R, Bissel K, Boillot F, Caminero J A, et al. Management of TB: a guide to the essentials of good practice. Paris: International Union against Tuberculosis and Lung Diseases; 2010

28. Global TB control: surveillance, planning, financing. Geneva: World Health Organization; 2007.

29. Oeltmann JE, Chengeta B, Mboya JJ, Wells CD, Kilmarx PH, Samandari T, et al. Reported childhood tuberculosis treatment outcomes, Gaborone and Francistown, Botswana, 1998-2002. Int J Tuberc Lung Dis. 2008 Feb;12(2):186-92. PMID:18230252

30. Lolekha R, Anuwatnonthakate A, Nateniyom S, Sumnapun S, Yamada N, Wattanaamornkiat W, et al. Childhood TB epidemiology and treatment outcomes in Thailand: a TB active surveillance network, 2004 to 2006. BMC Infect Dis. 2008;8(1):94. PMID:18637205

31. Global Tuberculosis Control. A short update to the 2009 report. Geneva: World Health Organization; 2009.

32. El Sony AI, Baraka O, Enarson DA, Bjune G. Tuberculosis control in Sudan against seemingly insurmountable odds. Int J Tuberc Lung Dis. 2000 Jul;4(7):657-64. PMID:10907769 